\title{
Building Physics during the Restoration of the Teatro Nuovo in Spoleto
}

\author{
Vincenzo Vodola \\ ${ }^{1}$ Department of Architecture, University of Bologna \\ Via Cavalcavia 61, 47521 Cesena, Italy \\ vincenzo.vodola2@unibo.it
}

\begin{abstract}
The Teatro Nuovo, the theatre where the famous international "Festival of the Two Worlds" is based, after a full restoration work and functional adaptation, has been returned to the city of Spoleto and its audience. The restoration works of the theatre started in 2003 for improving the safety levels and for adapting the operational functionality of the machines on the stage to the current needs of the show, including the acoustic performances. In particular, the Department of Energetic Engineering, Nuclear and Environmental Control of the University of Bologna collaborated with the Teatro Nuovo from the acoustic point of view,

In order to monitor the acoustic performance of the concert hall, two campaigns of acoustic measurements, respectively before and after the restoration works, have been conducted, verifying the conservation of its original acoustical characteristics. The measurements were performed through an omnidirectional loudspeaker, a dummy head and a Soundfield probe.

The results of the measurements of every campaign are presented in this article.
\end{abstract}

Keywords: Acoustical design, Acoustic spatialisation, Room acoustics measurements.

\section{Introduction}

Evaluating building performance is a central topic to align the building to the overcoming needs and for complying with Energy efficiency legislation [1]. In-situ measurement are of great interest since they help designing scenarios [2] and test the simulated ones [3]. Furthermore, new metrics such as exergy [4] are adopted especially in Research \& Development plans for innovative solutions [5] in the field of energy [6], acoustics [7] and comfort [8]. Great attention in the last decade was given to energy involving buildings at different scales, from the single one to urban level [9] but neglecting the main aspects of indoor wellbeing [10] mainly related to thermal insulation layers [11] and acoustic materials used in the ambient [12]. Experimental campaign is crucial to check the effective benefits of the designed solution once become real with a focus on cutting-edge technologies [13] installations, changes in the systems due to the novel components [14], performance outside the building's boundaries such as emissions [15] or energy exchanges with the Grid [16] and inside the building like the occupants' behaviour [17]. When the analysis is related to an existing building, its architectural features and its main past and future used must be considered. In the case of a theatre, acoustics is the main building physics matter to study and to check once the renovation is ended [18]. This paper deals with the case study of a theatre in Spoleto, Italy. It is the Teatro Nuovo, erected between 1853 and 1864, based on the plan of the architect Ireneo Oleandri: it was realised starting from the necessity to construct a new theatre, in a central place of the town of Spoleto, which could replace the previous "Ancient theatre", considered narrow, echoless and filthy.

The building represents a remarkable example of neoclassic architecture and of the nineteenth-century theatre "in Italian style" (see Figure 1), and nowadays it hosts the Festival of the Two Worlds, the Lyric Experimental Theatre and other cultural events. The adaptation and restoration works of the Teatro Nuovo, lasting 3 and half years, have allowed to improve the functionality of the theatre as a whole, to adjust the parts not in compliance with norms regarding the conditions of emergency of the public and of the structure. The design and the modelling part had to guarantee a correct use of the structures in the respect of the original characteristics of the architecture, to adapt the operation of the stage machines to current needs of the show and to enhance the levels of comfort for the public, including the acoustics. Here the link to be verified between foreseen performance and actual ones after restoration is important [19]. The new adjustments requires a deep investigation on the energy supply even considering the option to leave the terminals once already part of the listed interior design and, therefore, not to be modified [20]. Anyway, a detail will be given only to acoustics. 


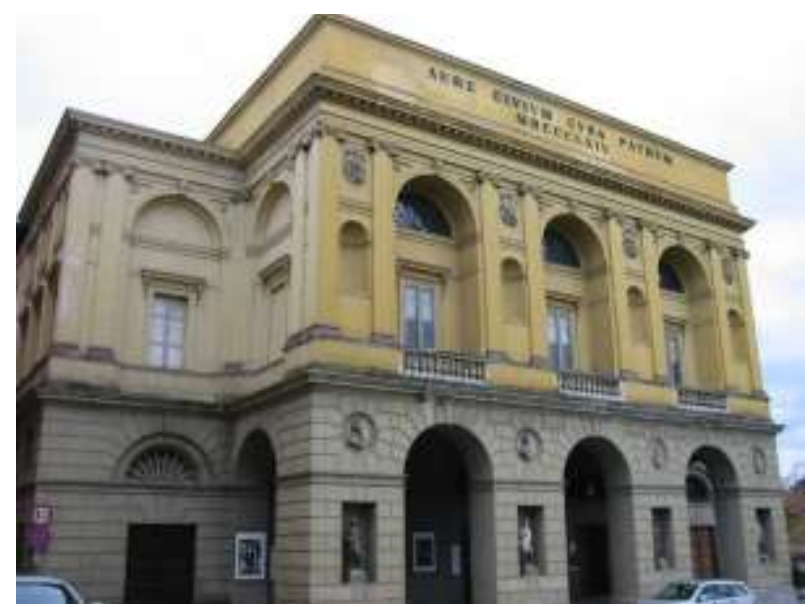

Fig. 1: Front of the Teatro Nuovo.

The works allowed to consolidate the wooden structures of the boxes, to improve the conditions of emergency for the workers and the public through the adaptation of the parapets in the boxes and to restore the pictorial and plastic decorations of the "Show Hall", of the "XVII September Hall". Referring to the façades, a reconstruction of the flooring in the arcade and in the entrance halls occurred. Since the restoration works required a special care both from the conservation of the original characteristics of the architecture of the "Show Hall" point of view and of its acoustic functioning, the Umbria Region commissioned the Department of Energetic Engineering, Nuclear and Environmental Control of the University of Bologna the acoustic consultancy since its experience [21-23]. Before closing the theatre, a first campaign of acoustic measurement has been carried out, and once the Theatre reopened, a second campaign was carried out, in order to control any modification of the acoustic performance. In this article the two campaigns of acoustic measurement, carried out before and after the restoration, are analysed and compared.

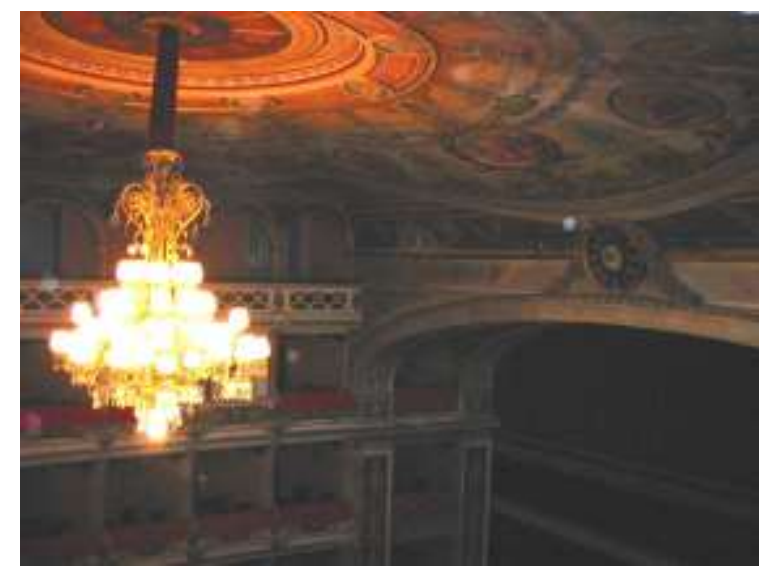

Fig. 2: View of the chandelier and the clock of the Teatro Nuovo.

\section{Measurements of Acoustical Quality}

The measurements were conducted for every positioning the loudspeakers in the stage-proscenium, and microphones in several points in the stage.

For both two campaign of acoustic measurements the following instruments were utilized:

- a dummy head (Sennheiser), which allowed the measurements of binaural parameters, like IACC;

- an equalised, omnidirectional, loudspeaker located in few positions on the stage;

- a Soundfield microphone (MK V) which was added to the dummy head for the measurements of 3dimensional parameters like LE and LF, and also allowed the measurements of mono-aural parameters, like reverberation time, clarity and centre time. 
The measurements were conducted by means of a logarithmic sinusoidal sweep, ranging between $40 \mathrm{~Hz}$ to $20 \mathrm{kHz}$. The signals acquired by the microphone were stored in the 20 bits, $96 \mathrm{kHz}$ soundboard (Layla) of the computer, and then post processed in the laboratory.

\section{Analysis of Measurements}

The Teatro Nuovo consists of a shoe-box shape and four orders of balconies (see Figure 3).

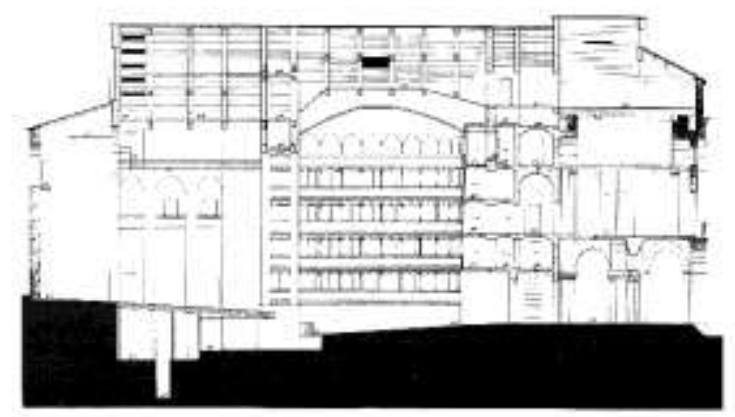

Fig. 3: Longitudinal view of the theatre.

The acoustical measurements were performed, in the first campaign of measurements in 2002, by positioning the sound source in 2 positions and the microphones in 54 positions between the stage and balcony, while in the second campaign in 2007, measurements are carried out by the sound source in 4 positions and the microphones in 66 positions between the stage and balcony. This could allow the opportunity to test innovative materials [24] and to build mathematical models to investigate deeply the acoustic phenomena [25] or simulation tool to check performance [26], even in historical buildings [27].

\subsection{Frequency Analysis of Acoustical Parameters}

As mentioned, the acoustic parameters were calculated from the omnidirectional (W channel) output of Soundfield microphone, whilst LE and LF were calculated combining the W and Y tracks of the Soundfield. The frequency response of the theatre could be evaluated analysing in the frequency domain the acoustical parameters. All acoustical parameters were calculated both in the stalls and for the balcony for every campaign. In figure 4 a) and b) the histograms in the frequency domain of EDT and $\mathrm{T}_{20}$ are depicted, whilst in figure $5 \mathrm{a}$ ) and b) the frequency values of $\mathrm{C}_{50}$ and $\mathrm{C}_{80}$ are reported. Comparing both the Early Decay Time and $\mathrm{T}_{20}$ measured in the two campaigns, it is evidence that the theatre presents values rather low of reverberation and gradually reduction of reverberation time up to high frequencies. The Teatro Nuovo resulted a rather dry theatre as many other Italian theatres. Since the optimal values for $\mathrm{T}_{20}$ ranges, for the opera, between 1 and $1.5 \mathrm{~s}$, and for symphonic music between $1.8 \mathrm{~s}$ and $2.6 \mathrm{~s}$, the measurements highlighted the lyric aptitude of the Teatro Nuovo, especially at medium frequencies. Comparing the values reported in figure $5 \mathrm{a}$ ) and b), there is evidence, on the contrary, that the restoration work has modified the indexes of clarity, rather that the reverberation time. In 2002 the values of $\mathrm{C}_{80}$ resulted too high, with values for opera houses not acceptable. On the other hand, the values of $\mathrm{C}_{50}$, variable between $-2 \mathrm{~dB}$ and $2 \mathrm{~dB}$ particularly at low and medium frequencies, demonstrated that the theatre is now more appropriate for speech. On the other hand, the measurements conducted in 2007 has revealed that $C_{50}$, resulted rather high, while the $C_{80}$, ranging between $-2 \mathrm{~dB}$ and $2 \mathrm{~dB}$ for almost all frequencies, demonstrates that the Teatro Nuovo is suitable for musical performances, as previously shown by $\mathrm{T}_{20}$. 

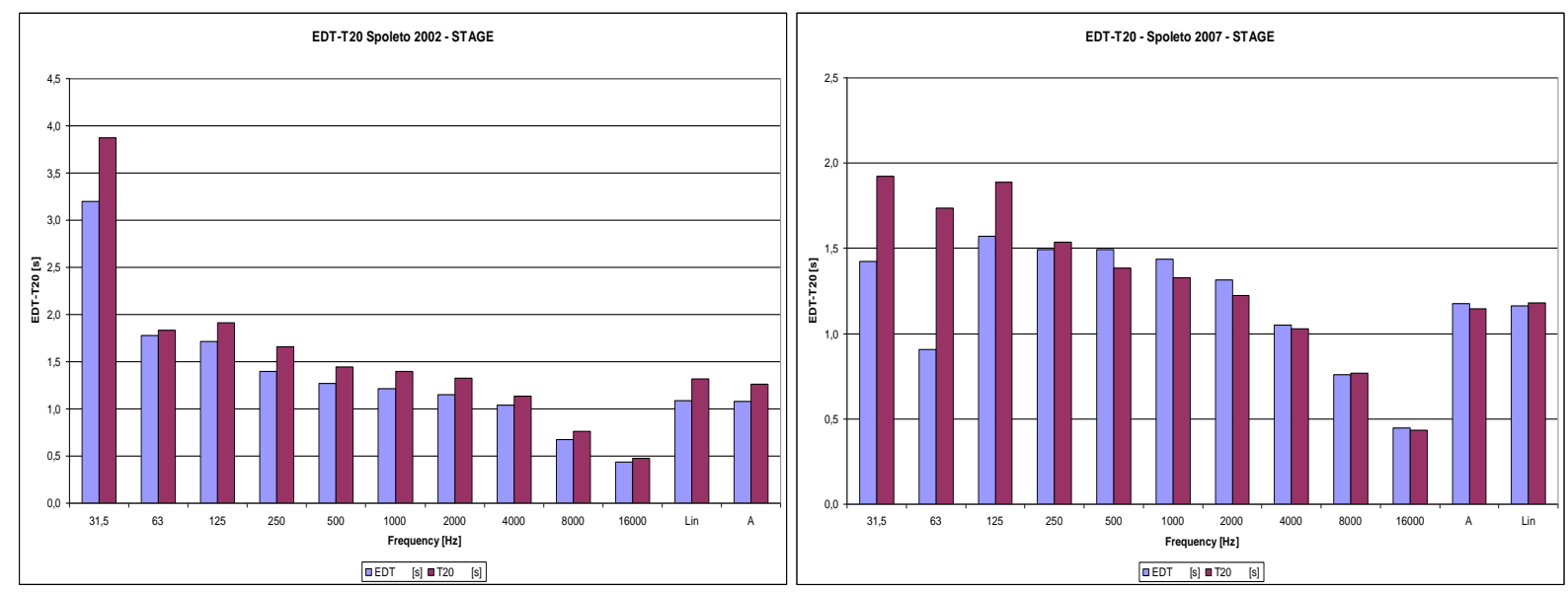

Fig. 4: EDT-T20- Teatro Nuovo 2002-stage (a) and 2007-stage (b).
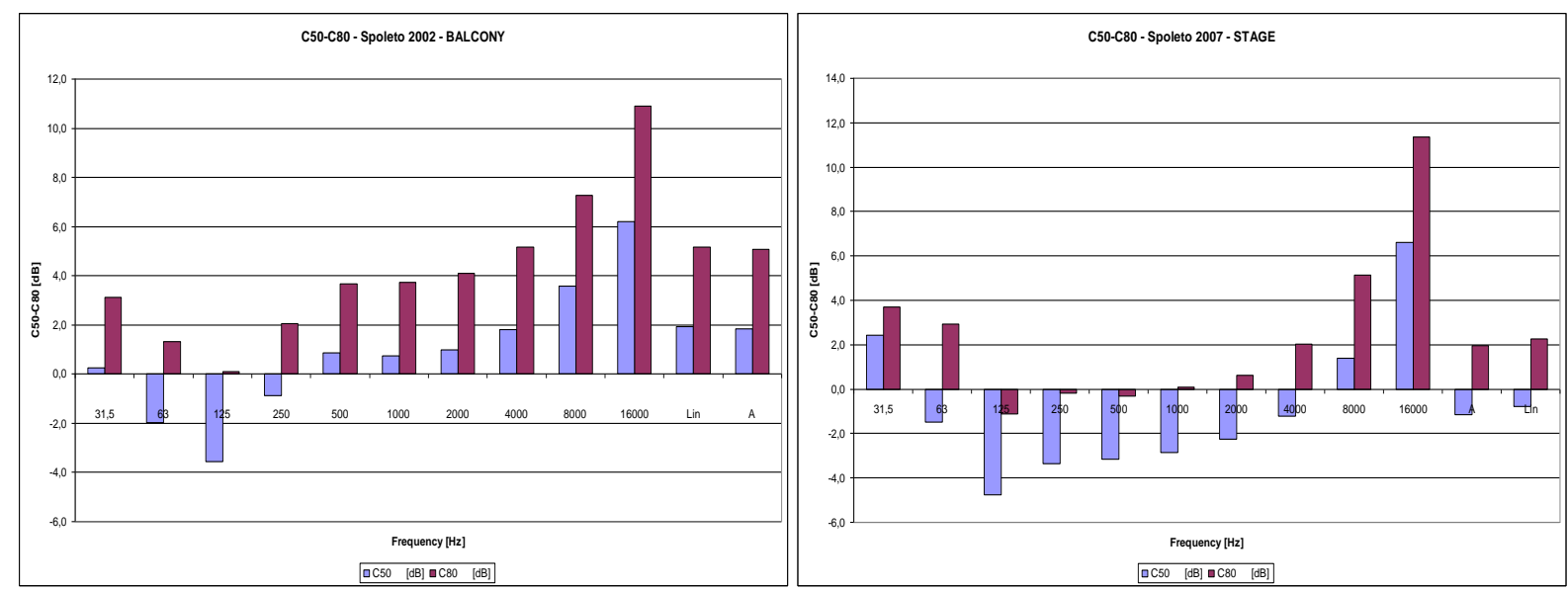

Fig. 5: $\mathrm{C}_{50}-\mathrm{C}_{80}$ - Teatro Nuovo 2002-stage (a) and 2007-stage (b).

\subsection{Frequency Analysis of Acoustical Parameters}

From the measurements, the more important spatial acoustical parameters (IACC, LE and LF) were calculated.

The Inter Aural Cross-Correlation coefficient (IACC) is defined as [1]:

$$
I A C C=\left|\rho(\tau)_{\max }\right|,(\tau \leq 1 \mathrm{~ms})
$$

where:

$$
\rho(\tau)=\frac{\phi_{d s}(\tau)}{\sqrt{\phi_{d}^{2}(0)} \cdot \sqrt{\phi_{s}^{2}(\tau)}}
$$

The spatial acoustical maps, which describe the distribution inside the theatre, of IACC are reported in Figure 8 for every campaign of acoustic measurement. Considering the spatial distribution of sound energy, the maps of IACC in the Teatro Nuovo, before the restoration (Figure 6a), compared with the maps after the works (Figure 6b), reveals an increasing value of IACC, whose value moved from 0.3 to 0.5 . 


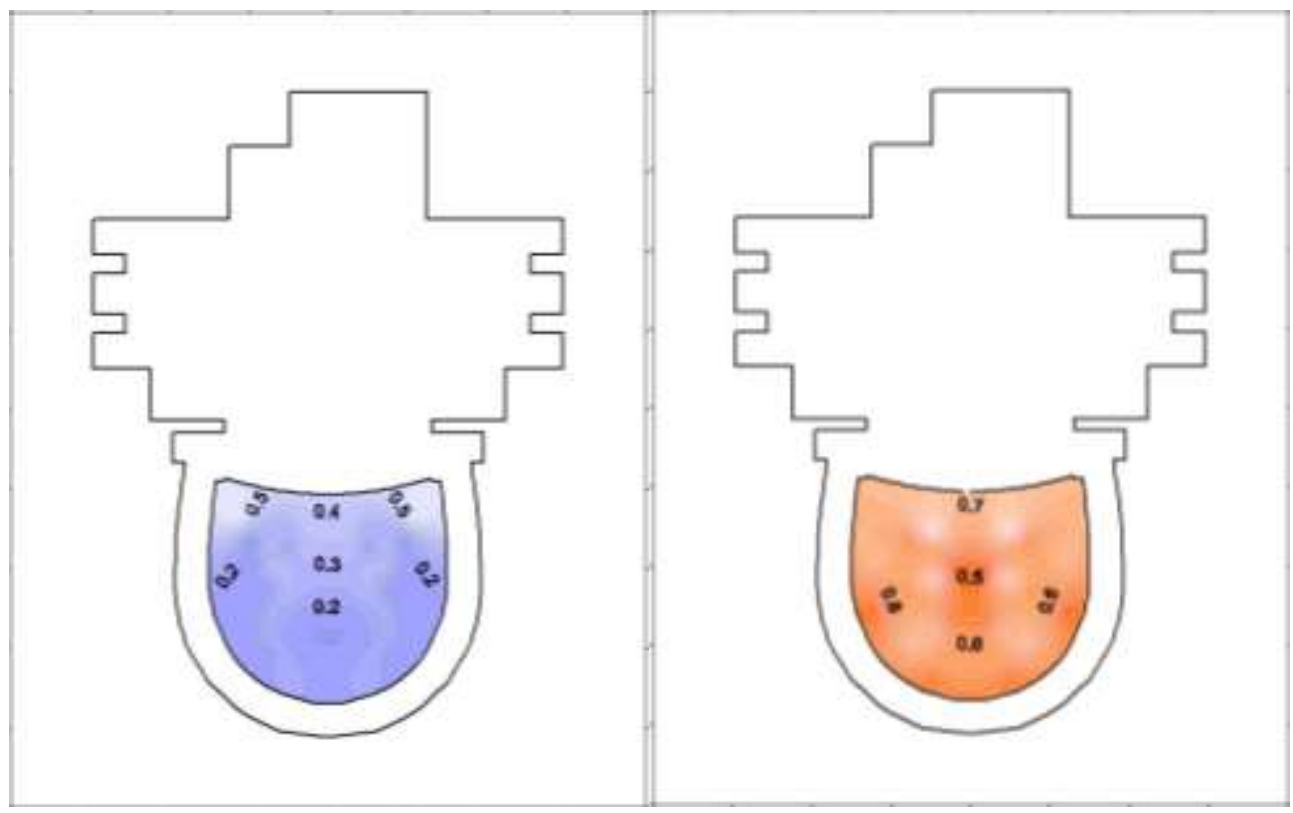

Fig. 6: IACC- Teatro Nuovo 2002-stage (a) and 2007-stage (b).

\section{Conclusion}

In this work the acoustical properties of Spoleto's Teatro Nuovo have been analysed, comparing the two different campaigns of acoustic measurements conducted in 2002 and in 2007. The restoration works of the theatre were necessary to increase the safety of the theatre and to adapt the operational functionality of the machines on the stage to the current needs of the show. The two campaigns of acoustic measurements have been carried out to check the acoustic performance of the theatre, before and after the works. The comparison has shown some slight modification of the acoustic characteristics. The acoustic performances of the theatre are globally enhanced, contributing at good perception of acoustic space, especially for the opera and symphonic music. Today, the Teatro Nuovo can, therefore, gives again hospitality to Festival of the Two Worlds with a renewed form.

\section{Acknowledgements}

The Author acknowledge prof. Lamberto Tronchin for his fundamental help during the acoustic measurements and evaluation of the results. This paper was partially funded within the project "Research for SEAP: a platform for municipalities taking part in the Covenant of Mayors", financed by Italian Government in the framework of PRIN 2015.

\section{References}

[1] L. De Santoli, F. Mancini, B. Nastasi and S. Ridolfi, "Energy retrofitting of dwellings from the 40's in Borgata Trullo - Rome," Energy Proc, vol. 133, pp. 281-289, 2017.

[2] M. Manfren and B. Nastasi, "From in-situ measurement to regression and time series models: An overview of trends and prospects for building performance modelling," AIP Conference Proceedings, 2019.

[3] L.C. Tagliabue, M. Manfren, A.L.C. Ciribini and E. De Angelis, "Probabilistic behavioural modeling in building performance simulation-The Brescia eLUX lab," Energy Build, vol. 128, pp. 119-131, 2016.

[4] K. Fabbri and L. Tronchin, "Analysis of buildings' energy consumption by means of exergy method," Int. J. of Exergy, vol. 5, no. 5-6, pp. 605-625, 2008

[5] B. Nastasi, "Hydrogen Policy, Market and R\&D Projects" in Hydrogen Production from Solar Energy, F.Calise et al., Ed. Amsterdam: Elsevier, 2019.

[6] A. L. C. Ciribini, D. Pasini, L. C. Tagliabue, M. Manfren, B. Daniotti, S. Rinaldi and E. De Angelis 'Tracking Users' Behaviors through Real-time Information in BIMs: Workflow for Interconnection in the Brescia Smart Campus Demonstrator," Proc Eng, vol. 180, pp. 1484-1494, 2017. 
[7] L. Tronchin, "Modal analysis and intensity of acoustic radiation of the kettledrum," J. Acoust. Soc. Am., vol. 117, no. 2, pp. 926-933, 2005.

[8] F. Scamoni, E. A. Piana and C. Scrosati, "Experimental evaluation of the sound absorption and insulation of an innovative coating through different testing methods," Build. Acous., vol. 24, no. 3, pp. 173-191, 2017.

[9] B. Nastasi, G. Lo Basso, D. A. Garcia, F. Cumo and L. De Santoli, "Power-to-gas leverage effect on power-to-heat application for urban renewable thermal energy systems," Int. J. of Hydrogen En., vol. 43, pp. 23076-23090, 2018.

[10] K. Fabbri and L. Tronchin, "Indoor environmental quality in low energy buildings," Energy Proc., vol. 78, pp. 27782783, 2015.

[11] U. Berardi, L. Tronchin, M. Manfren and B. Nastasi, "On the effects of variation of thermal conductivity in buildings in the Italian construction sector," Energies, vol. 11, no. 4, 872, 2018.

[12] L. Tronchin, "On the acoustic efficiency of road barriers: The reflection index," Int. J. Mechanics, vol. 7, no. 3, pp. 318-326, 2013.

[13] B. Castellani, E. Morini, B. Nastasi, A. Nicolini and F. Rossi, "Small-scale compressed air energy storage application for renewable energy integration in a listed building" Energies, vol. 11, no. 7, 1921, 2017.

[14] L. Tronchin, M. Manfren, V. Vodola and B. Nastasi, "Building performance monitoring: from in-situ measurement to regression-based approaches," Journal of Physics: Conference Series, 2019.

[15] B. Castellani, S. Rinaldi, E. Morini, B. Nastasi, and F. Rossi, "Flue Gas Treatment by Power-to-Gas Integration for Methane and Ammonia Synthesis - Energy and Environmental Analysis," Energy Conv Manage, vol. 171, pp 626634, 2018.

[16] L. Tronchin, M. Manfren and B. Nastasi, "Energy analytics for supporting built environment decarbonisation," Energy Proc, vol. 157, pp. 1486-1493, 2019.

[17] A. Zani, L.C. Tagliabue, T. Poli, A.L.C. Ciribini, E. De Angelis and M. Manfren, "Occupancy Profile Variation Analyzed through Generative Modelling to Control Building Energy Behavior," Proc Eng, vol. 180, pp. 1495-1505, 2017.

[18] L. Tronchin, "Francesco Milizia (1725-1798) and the acoustics of his Teatro Ideale (1773)," Acta Acustica United Acustica, vol. 99, no. 1, pp. 91-97, 2013.

[19] L. Tronchin, M. Manfren and P.A. James, "Linking design and operation performance analysis through model calibration: Parametric assessment on a Passive House building," Energy, vol. 165, pp. 26-40, 2018.

[20] L. De Santoli, G. Lo Basso and B. Nastasi, "Innovative Hybrid CHP systems for high temperature heating plant in existing buildings," Energy Proc, vol. 133, pp. 207-218, 2017.

[21] A. Farina and L. Tronchin "On the "Virtual" reconstruction of sound quality of trumpets," Acustica, vol. 86, no. 4, pp. 737-745, 2000.

[22] A. Farina and L. Tronchin, "3D sound characterisation in theatres employing microphone arrays," Acta Acustica United Acustica, vol. 99, no. 1, pp. 118-125, 2013.

[23] L. Tronchin and V. L. Coli "Further investigations in the emulation of nonlinear systems with Volterra series," AES J. Audio Eng. Soc., vol. 63, no. 9, pp. 671-683, 2015.

[24] E.A. Piana, C. Petrogalli, D. Paderno and U. Carlsson "Application of the Wave Propagation Approach to Sandwich Structures: Vibro-Acoustic Properties of Aluminum Honeycomb Materials," Appl. Sci., vol. 8, no. 1, 45, 2018.

[25] R. Shimokura, L. Tronchin, A. Cocchi and Y. Soeta, "Subjective diffuseness of music signals convolved with binaural impulse responses," J. of Sound Vibration, vol. 330, no. 14, pp. 3526-3537, 2011.

[26] F. Re Cecconi, M. Manfren, L.C. Tagliabue, A. L. C. Ciribini and E. De Angelis, "Probabilistic behavioral modeling in building performance simulation: A Monte Carlo approach,” Energy Build, vol. 148, pp. 128-141, 2017.

[27] L. Tronchin and K. Fabbri, "Energy and microclimate simulation in a heritage building: Further studies on the Malatestiana Library," Energies, vol. 10, no. 10, pp. 1621, 2017 